\title{
On the uniqueness of viscosity solutions for first order partial differential-functional equations
}

\author{
by KRzyszTof Topolski (Gdańsk)
}

\begin{abstract}
We consider viscosity solutions for first order differential-functional equations. Uniqueness theorems for initial, mixed, and boundary value problems are presented. Our theorems include some results for generalized ("almost everywhere") solutions.
\end{abstract}

1. Introduction. Let $T>0, \tau_{0} \in \mathbb{R}_{+}$and $\tau \in \mathbb{R}_{+}^{n}\left(\mathbb{R}_{+}=[0, \infty)\right)$ be given constants, and $\Omega \subset \mathbb{R}^{n}$ any open domain. Define $\Omega_{\tau}=\left\{x \in \mathbb{R}^{n}\right.$ : $\operatorname{dist}(x, \Omega)<\tau\}, \partial_{0} \Omega=\Omega_{\tau} \backslash \Omega, \Theta=(0, T) \times \Omega, \Theta_{0}=\left(-\tau_{0}, 0\right] \times \Omega_{\tau}, \partial_{0} \Theta=$ $(0, T) \times \partial_{0} \Omega$ and $E=\Theta \cup \Theta_{0} \cup \partial_{0} \Theta$. Let $B=\left\{x \in \mathbb{R}^{n}:\|x\| \leq \tau\right\}$ and $D=\left[-\tau_{0}, 0\right] \times B$. For every $z: E \rightarrow \mathbb{R}$ and $(t, x) \in \Theta$ we define a function $z_{\langle t, x\rangle}: D \rightarrow \mathbb{R}$ by $z_{\langle t, x\rangle}(s, y)=z(t+s, x+y),(s, y) \in D$. If $z: \Omega_{\tau} \rightarrow \mathbb{R}$ and $x \in \Omega$, we define $z_{\langle x\rangle}: B \rightarrow \mathbb{R}$ by $z_{\langle x\rangle}(y)=z(x+y), y \in B$.

For every metric space $X$ we denote by $C(X)$ the class of all continuous functions from $X$ into $\mathbb{R}$ and by $\operatorname{BUC}(X)$ the class of all uniformly continuous and bounded functions from $X$ into $\mathbb{R}$. Let $W^{1 \infty}(X)$ and $W_{\text {loc }}^{1 \infty}(X)$ be the classes of all Lipschitz and locally Lipschitz functions from $X$ into $\mathbb{R}$. Recall that $u \in W^{1 \infty}(X)$ (resp. $\left.W_{\text {loc }}^{1 \infty}(X)\right) \Leftrightarrow D u$ exists a.e. and is bounded (resp. locally bounded). For $f_{m}, f \in C(X), m \in \mathbb{N}, f_{m} \rightarrow f$ means uniform convergence on compact subsets of $X$.

Let $H: \Theta \times C(D) \times \mathbb{R}^{n} \rightarrow \mathbb{R}$ (resp. $H: \Omega \times \mathbb{R} \times C(B) \times \mathbb{R}^{n} \rightarrow \mathbb{R}$ ) be a continuous function of the variables $(t, x, w, p)$ (resp. $(x, r, w, p))$, and let $g \in C\left(\Theta_{0} \cup \partial_{0} \Theta\right)$ and $f \in C\left(\partial_{0} \Omega\right)$ be given functions.

We consider two classes of differential-functional equations: the initialboundary value problem

$$
\begin{array}{ll}
D_{t} z(t, x)+H\left(t, x, z_{\langle t, x\rangle}, D_{x} z(t, x)\right)=0 & \text { in } \Theta, \\
z(t, x)=g(t, x) & \text { in } \Theta_{0} \cup \partial_{0} \Theta,
\end{array}
$$

1991 Mathematics Subject Classification: 35D99, 35B30.

Key words and phrases: uniqueness, viscosity solution, differential-functional equation, almost everywhere solution. 
and the boundary value problem

$$
\begin{array}{ll}
H\left(x, z(x), z_{\langle x\rangle}, D z(x)\right)=0 & \text { in } \Omega, \\
z(x)=g(x) & \text { in } \partial_{0} \Omega .
\end{array}
$$

Even though we called $(\mathrm{C})$ "the initial-boundary value problem", it is an initial value problem for $\Theta=(0, T) \times \mathbb{R}^{n}$.

Definition 1. A function $u \in C(E)$ is a viscosity subsolution (resp. supersolution) of $(\mathrm{C})$ provided for all $\varphi \in C^{1}(\Theta)$ if $u-\varphi$ attains a local maximum (resp. minimum) at $\left(t_{0}, x_{0}\right) \in \Theta$ then

$$
\begin{gathered}
D_{t} \varphi\left(t_{0}, x_{0}\right)+H\left(t_{0}, x_{0}, u_{\left\langle t_{0}, x_{0}\right\rangle}, D_{x} \varphi\left(t_{0}, x_{0}\right)\right) \leq 0 \\
\left(\operatorname{resp.} D_{t} \varphi\left(t_{0}, x_{0}\right)+H\left(t_{0}, x_{0}, u_{\left\langle t_{0}, x_{0}\right\rangle}, D_{x} \varphi\left(t_{0}, x_{0}\right)\right) \geq 0\right)
\end{gathered}
$$

and

$$
\begin{gathered}
u(t, x) \leq g(t, x) \quad \text { in } \partial_{0} \Theta \\
\text { (resp. } \left.u(t, x) \geq g(t, x) \text { in } \partial_{0} \Theta\right) .
\end{gathered}
$$

A function $u \in C(E)$ is a viscosity solution of (1) if $u$ is both a viscosity subsolution and supersolution of (1).

Since for the problem (D) the definition is parallel, we will not write it out.

We denote by $\operatorname{SUB}(X, g), \operatorname{SUP}(X, g)$ (or $\operatorname{SUB}(X, H, g), \operatorname{SUP}(X, H, g)$ when $H$ is not clear) the sets of all viscosity subsolutions and supersolutions of the problem (C) and (D), with $X=E$ or resp. $X=\Omega_{\tau}$.

This notion of solution was first introduced by M. G. Crandall and P. L. Lions in [3] and developed in [2], [9].

As it is well known that classical solutions exist only locally, new notions of solutions (weak solutions, "almost everywhere" solutions, viscosity solutions) are introduced in order to obtain global existence results. The problem which naturally arises here is their uniqueness. The literature on this subject is extensive. We only mention some review papers.

The uniqueness of classical solutions for first order partial differential equations was considered by V. Lakshmikantham and S. Leela [7] and by J. Szarski [10]. The same problem for functional-differential equations was investigated by Z. Kamont [4] and by A. Augustynowicz and Z. Kamont [1]. The uniqueness of "almost everywhere" solutions was studied by S. N. Kruzhkov [5], [6]. The functional case was treated by H. Leszczyński [8].

For $q=\left(q_{1}, \ldots, q_{n}\right) \in \mathbb{R}^{n}$ we write $\|q\|$ for the Euclidean norm. Let $\|w\|_{X}$ denote the supremum norm of $w \in C(X), \omega_{w}(\varepsilon)$ the modulus of continuity and $w^{+}=\max (w, 0)$.

2. The initial-boundary value problem. In this section we consider the problem $(\mathrm{C})$. 
We will need the following

Assumption $\mathrm{H}_{1}$. (i) The function $H: \Theta \times K_{1} \times K_{2} \rightarrow \mathbb{R}$ is uniformly continuous for any bounded sets $K_{1} \subset C(D), K_{2} \subset \mathbb{R}^{n}$.

(ii) For every $R \geq 0$,

$\lim _{\alpha \rightarrow 0} \sup \{|H(t, x, w, p)-H(s, y, w, p)|$ :

$$
\left.|x-y|<\alpha,|t-s|<\alpha,|x-y||p|<\alpha,\|w\|_{D} \leq R\right\}=0 .
$$

(iii) There exists a constant $C>0$ such that

$$
H(t, x, w, p)-H(t, x, \bar{w}, p) \geq-C\left\|(w-\bar{w})^{+}\right\|_{D} .
$$

The following is easy to check:

R e mark 1. The condition (iii) holds if and only if $H$ is nondecreasing and satisfies the Lipschitz condition with respect to $w$.

THEOREM 1. Suppose that Assumption $\mathrm{H}_{1}$ is satisfied and $u, v \in$ $\operatorname{BUC}(E), u \in \operatorname{SUB}(E, f), v \in \operatorname{SUP}(E, g)$, where $f, g$ are continuous. Then

$$
\left\|(u-v)^{+}\right\|_{E} \leq \exp (C T)\left\|(f-g)^{+}\right\|_{\Theta_{0} \cup \partial_{0} \Theta} .
$$

(*) Moreover, if $u, v \in W^{1 \infty}(E)$ then the condition (ii) of $\mathrm{H}_{1}$ is not necessary.

The proof of the theorem is based on the following

Proposition 1. Let $a>0$ and $h, F \in C([0, a])$. Assume that $h$ is a viscosity solution of $h^{\prime} \leq F$ (i.e. $h$ is a viscosity subsolution of $h^{\prime}=F$ ) in $(0, a)$. Then

$$
h(t) \leq h(s)+\int_{s}^{t} F(\nu) d \nu \quad \text { for } 0 \leq s \leq t \leq a .
$$

The proof can be found in [3], p. 12 .

Proof of Theorem 1. Define

$$
\begin{aligned}
M(t) & =\sup \{u(s, x)-v(s, x):(s, x) \in \Theta[t]\}, \\
M_{0}(t) & =\sup \left\{u(s, x)-v(s, x):(s, x) \in\left(\Theta_{0} \cup \partial_{0} \Theta\right)[t]\right\}, \\
\bar{M}(t) & =\sup \{u(s, x)-v(s, x):(s, x) \in E[t]\},
\end{aligned}
$$

where $G[t]=\left\{(s, x) \in G:-\tau_{0}<s \leq t\right\}$ for $G \subset \mathbb{R}^{n+1}$.

If $M(T) \leq M_{0}(T)$ the proof is complete. Suppose that $M(T)>M_{0}(T)$. Since $M(0) \leq M_{0}(0)$ there exists $0 \leq t_{*}<T$ such that

(5) $\quad M(t)>M_{0}(t)$ for $t \in\left(t_{*}, T\right]$ and $M\left(t_{*}\right)=M_{0}\left(t_{*}\right)=\bar{M}\left(t_{*}\right)$.

Let $\eta \in C^{1}\left(\left(t_{*}, T\right)\right)$, and suppose $M-\eta$ attains its local maximum at some point $t_{0} \in\left(t_{*}, T\right)$. Since $M$ is nondecreasing it is clear that $\eta^{\prime}\left(t_{0}\right) \geq 0$. We need to consider two cases: $\eta^{\prime}\left(t_{0}\right)>0$ and $\eta^{\prime}\left(t_{0}\right)=0$. 
Let $\eta^{\prime}\left(t_{0}\right)>0$. It follows from Lemma 1.4 of [3] that we can find a nondecreasing function $\bar{\eta} \in C^{1}\left(\left[t_{*}, T\right]\right)$ such that $\bar{\eta}^{\prime}\left(t_{0}\right)=\eta^{\prime}\left(t_{0}\right)$ and $(M-\bar{\eta})\left(t_{0}\right)>(M-\bar{\eta})(t)$ for $t \neq t_{0}$. To simplify the notation write $\eta=\bar{\eta}$.

Let $(t, s, x, y) \in\left[t_{*}, T\right] \times\left[t_{*}, T\right] \times \bar{\Omega} \times \bar{\Omega}, \mathcal{M}=\max \left(\|u\|_{E},\|v\|_{E}\right)$ and $N=\sup \eta$. Put

(6) $\Psi(t, s, x, y)=u(t, x)-v(s, y)-\eta\left(\frac{t+s}{2}\right)+K \phi_{\alpha}(t-s) \psi_{\alpha}(x-y)$

where $K=5 \mathcal{M}+2 N, \phi_{\alpha}(\cdot)=\phi(\cdot / \alpha), \psi_{\alpha}(\cdot)=\psi(\cdot / \alpha)$ and $\phi \in C^{1}(\mathbb{R}), \psi \in$ $C^{1}\left(\mathbb{R}^{n}\right), 0 \leq \phi, \psi \leq 1, \phi(0)=\psi(0)=1, \operatorname{supp} \phi \subset[-1,1], \operatorname{supp} \psi \subset B(0,1)$, $\phi, \psi$ are radial decreasing and for $r^{2} \leq 1 / 2,\|z\|^{2} \leq 1 / 2, \phi(r)=1-r^{2}$, $\psi(z)=1-\|z\|^{2}$.

Let $\delta>0$ and let $\left(t^{\prime}, s^{\prime}, x^{\prime}, y^{\prime}\right)$ be such that $\Psi\left(t^{\prime}, s^{\prime}, x^{\prime}, y^{\prime}\right)>\sup \Psi-\delta$. Put

$$
\Phi(t, s, x, y)=\Psi(t, s, x, y)+2 \delta \xi(x, y)
$$

where $\xi \in C^{1}\left(\mathbb{R}^{n} \times \mathbb{R}^{n}\right), 0 \leq \xi \leq 1, \xi\left(x^{\prime}, y^{\prime}\right)=1$, supp $\xi \subset B\left(\left(x^{\prime}, y^{\prime}\right), 1\right)$ and $\|D \xi\|<k$ for $k>0$ independent of $\alpha, \delta$. Since $\Phi(t, s, x, y) \leq \sup \Psi$ for $\left|x-x^{\prime}\right|^{2}+\left|y-y^{\prime}\right|^{2} \geq 1$ and $\Phi\left(t^{\prime}, s^{\prime}, x^{\prime}, y^{\prime}\right)>\sup \Psi+\delta$ it follows that $\Phi$ attains its global maximum at some point $\left(t_{\alpha \delta}, s_{\alpha \delta}, x_{\alpha \delta}, y_{\alpha \delta}\right)$. This yields $\Phi\left(t_{\alpha \delta}, s_{\alpha \delta}, x_{\alpha \delta}, y_{\alpha \delta}\right) \geq \Phi(t, s, x, y)$ and by $(6),(7)$,

$$
\phi_{\alpha}\left(t_{\alpha \delta}-s_{\alpha \delta}\right) \psi_{\alpha}\left(x_{\alpha \delta}-y_{\alpha \delta}\right) \geq \mathcal{M} /(5 \mathcal{M}+2 N)-2 \delta>0
$$

for $\delta$ small, which implies $\left|t_{\alpha \delta}-s_{\alpha \delta}\right|<\alpha$ and $\left\|x_{\alpha \delta}-y_{\alpha \delta}\right\|<\alpha$. Moreover, since

$$
\Phi\left(t_{\alpha \delta}, s_{\alpha \delta}, x_{\alpha \delta}, y_{\alpha \delta}\right) \geq \Phi\left(t_{\alpha \delta}, t_{\alpha \delta}, x_{\alpha \delta}, x_{\alpha \delta}\right)
$$

we obtain by $(6),(7)$,

$$
\phi_{\alpha}\left(t_{\alpha \delta}-s_{\alpha \delta}\right) \psi_{\alpha}\left(x_{\alpha \delta}-y_{\alpha \delta}\right) \geq 1-\left(\omega_{v}(\alpha)+\omega_{\eta}(\alpha / 2)+2 \delta\right) / K .
$$

Recalling the definitions of $\phi_{\alpha}, \psi_{\alpha}$ we get $\left|t_{\alpha \delta}-s_{\alpha \delta}\right|<\alpha r(\alpha)$ and $\left|x_{\alpha \delta}-y_{\alpha \delta}\right|<\alpha r(\alpha)$ for $\delta<\alpha$ small enough where $r(\alpha) \rightarrow 0$ as $\alpha \rightarrow 0$.

We can assume, taking a subsequence if necessary, that $t_{\alpha \delta} \rightarrow \bar{t} \in\left[t_{*}, T\right]$ as $\alpha \rightarrow 0$ uniformly with respect to $\delta<\alpha$. We claim that $\bar{t}=t_{0}$. Indeed, since

$$
\Phi\left(t_{\alpha \delta}, s_{\alpha \delta}, x_{\alpha \delta}, y_{\alpha \delta}\right) \geq \Phi(t, t, x, x) \quad \text { for } t \in\left[t_{*}, t_{0}\right], x \in \Omega,
$$

and $\phi_{\alpha \delta}\left(t_{\alpha \delta}-s_{\alpha \delta}\right) \rightarrow 1, \psi_{\alpha}\left(x_{\alpha \delta}-y_{\alpha \delta}\right) \rightarrow 1$ as $\alpha \rightarrow 0$, letting $\alpha \rightarrow 0$ we obtain, by (6), (7), (8) and uniform continuity of $v$,

$$
M(\bar{t})-\eta(\bar{t}) \geq u(t, x)-v(t, x)-\eta(t) \geq u(t, x)-v(t, x)-\eta\left(t_{0}\right)
$$


(the last inequality is a consequence of monotonicity of $\eta$ ). Because $M\left(t_{0}\right)>$ $M\left(t_{*}\right)$ this yields

$$
M(\bar{t})-\eta(\bar{t}) \geq M\left(t_{0}\right)-\eta\left(t_{0}\right),
$$

which by the definition of $\eta$ means that $\bar{t}=t_{0} \in\left(t_{*}, T\right)$.

Observe now that we may assume that $x_{\alpha \delta}, y_{\alpha \delta} \in \Omega$. Indeed, if $x_{\alpha \delta}, y_{\alpha \delta}$ $\rightarrow x_{0} \in \partial \Omega$ then letting $\alpha \rightarrow 0$ in (8) we obtain

$$
u\left(t_{0}, x_{0}\right)-v\left(t_{0}, x_{0}\right)-\eta\left(t_{0}\right) \geq u(t, x)-v(t, x)-\eta(t)
$$

for $t_{*} \leq t \leq t_{0}, x_{0} \in \partial_{0} \Omega, x \in \Omega$, and as a consequence

$$
u\left(t_{0}, x_{0}\right)-v\left(t_{0}, x_{0}\right) \geq u(t, x)-v(t, x),
$$

which yields $M_{0}\left(t_{0}\right) \geq M\left(t_{0}\right)$ and contradicts (5) since $t_{0} \in\left(t_{*}, T\right]$.

Define

$$
\begin{aligned}
\lambda(t, x)= & v\left(s_{\alpha \delta}, y_{\alpha \delta}\right)+\eta\left(\left(t+s_{\alpha \delta}\right) / 2\right) \\
& -K \phi_{\alpha}\left(t-s_{\alpha \delta}\right) \psi_{\alpha}\left(x-y_{\alpha \delta}\right)-2 \delta \xi\left(x, y_{\alpha \delta}\right), \\
\gamma(s, y)= & u\left(t_{\alpha \delta}, x_{\alpha \delta}\right)-\eta\left(\left(t_{\alpha \delta}+s\right) / 2\right) \\
& +K \phi_{\alpha}\left(t_{\alpha \delta}-s\right) \psi_{\alpha}\left(x_{\alpha \delta}-y\right)+2 \delta \xi\left(x_{\alpha \delta}, y\right) .
\end{aligned}
$$

Notice that

$$
\begin{aligned}
& u-\lambda \text { has a local maximum at }\left(t_{\alpha \delta}, x_{\alpha \delta}\right) \in\left(t_{*}, T\right) \times \Omega, \\
& v-\gamma \text { has a local minimum at }\left(s_{\alpha \delta}, y_{\alpha \delta}\right) \in\left(t_{*}, T\right) \times \Omega .
\end{aligned}
$$

Since

$$
\begin{aligned}
& D_{t} \lambda\left(t_{\alpha \delta}, x_{\alpha \delta}\right)=\frac{1}{2} \eta^{\prime}\left(\left(t_{\alpha \delta}+s_{\alpha \delta}\right) / 2\right)-K D_{t} \phi_{\alpha}\left(t_{\alpha \delta}-s_{\alpha \delta}\right) \psi_{\alpha}\left(x_{\alpha \delta}-y_{\alpha \delta}\right), \\
& D_{t} \gamma\left(s_{\alpha \delta}, y_{\alpha \delta}\right)=-\frac{1}{2} \eta^{\prime}\left(\left(t_{\alpha \delta}+s_{\alpha \delta}\right) / 2\right)-K D_{t} \phi_{\alpha}\left(t_{\alpha \delta}-s_{\alpha \delta}\right) \psi_{\alpha}\left(x_{\alpha \delta}-y_{\alpha \delta}\right), \\
& D_{x} \lambda\left(t_{\alpha \delta}, x_{\alpha \delta}\right)=-K \phi_{\alpha}\left(t_{\alpha \delta}-s_{\alpha \delta}\right) D_{x} \psi_{\alpha}\left(x_{\alpha \delta}-y_{\alpha \delta}\right)-2 \delta D_{x} \xi\left(x_{\alpha \delta}, y_{\alpha \delta}\right), \\
& D_{x} \gamma\left(s_{\alpha \delta}, y_{\alpha \delta}\right)=-K \phi_{\alpha}\left(t_{\alpha \delta}-s_{\alpha \delta}\right) D_{x} \psi_{\alpha}\left(x_{\alpha \delta}-y_{\alpha \delta}\right)+2 \delta D_{y} \xi\left(x_{\alpha \delta}, y_{\alpha \delta}\right),
\end{aligned}
$$

and $u, v$ are resp. a subsolution and supersolution of (1) in $\Theta \backslash \Theta\left[t_{*}\right]$, we obtain the inequalities

$$
\begin{aligned}
& D_{t} \lambda\left(t_{\alpha \delta}, x_{\alpha \delta}\right)+H\left(t_{\alpha \delta}, x_{\alpha \delta}, u_{\left\langle t_{\alpha \delta}, x_{\alpha \delta}\right\rangle}, p_{\alpha \delta}-2 \delta D_{x} \xi\left(x_{\alpha \delta}, y_{\alpha \delta}\right)\right) \leq 0, \\
& D_{t} \gamma\left(s_{\alpha \delta}, y_{\alpha \delta}\right)+H\left(s_{\alpha \delta}, y_{\alpha \delta}, v_{\left\langle s_{\alpha \delta}, y_{\alpha \delta}\right\rangle}, p_{\alpha \delta}+2 \delta D_{y} \xi\left(x_{\alpha \delta}, y_{\alpha \delta}\right)\right) \geq 0
\end{aligned}
$$

(where $p_{\alpha \delta}=-K \phi_{\alpha}\left(t_{\alpha \delta}-s_{\alpha \delta}\right) D_{x} \psi_{\alpha}\left(x_{\alpha \delta}-y_{\alpha \delta}\right)$ ), which imply

$$
\eta^{\prime}\left(\left(t_{\alpha \delta}+s_{\alpha \delta}\right) / 2\right)+A_{\alpha \delta}+B_{\alpha \delta}+C_{\alpha \delta} \leq 0
$$

where

$$
\begin{aligned}
A_{\alpha \delta}= & H\left(t_{\alpha \delta}, x_{\alpha \delta}, u_{\left\langle t_{\alpha \delta}, x_{\alpha \delta}\right\rangle}, p_{\alpha \delta}-2 \delta D_{x} \xi\left(x_{\alpha \delta}, y_{\alpha \delta}\right)\right) \\
& -H\left(s_{\alpha \delta}, y_{\alpha \delta}, u_{\left\langle t_{\alpha \delta}, x_{\alpha \delta}\right\rangle}, p_{\alpha \delta}-2 \delta D_{x} \xi\left(x_{\alpha \delta}, y_{\alpha \delta}\right)\right), \\
B_{\alpha \delta}= & H\left(s_{\alpha \delta}, y_{\alpha \delta}, u_{\left\langle t_{\alpha \delta}, x_{\alpha \delta}\right\rangle}, p_{\alpha \delta}-2 \delta D_{x} \xi\left(x_{\alpha \delta}, y_{\alpha \delta}\right)\right) \\
& -H\left(s_{\alpha \delta}, y_{\alpha \delta}, v_{\left\langle s_{\alpha \delta}, y_{\alpha \delta}\right\rangle}, p_{\alpha \delta}-2 \delta D_{x} \xi\left(x_{\alpha \delta}, y_{\alpha \delta}\right)\right),
\end{aligned}
$$




$$
\begin{aligned}
C_{\alpha \delta}= & H\left(s_{\alpha \delta}, y_{\alpha \delta}, v_{\left\langle s_{\alpha \delta}, y_{\alpha \delta}\right\rangle}, p_{\alpha \delta}-2 \delta D_{x} \xi\left(x_{\alpha \delta}, y_{\alpha \delta}\right)\right) \\
& -H\left(s_{\alpha \delta}, y_{\alpha \delta}, v_{\left\langle s_{\alpha \delta}, y_{\alpha \delta}\right\rangle}, p_{\alpha \delta}+2 \delta D_{y} \xi\left(x_{\alpha \delta}, y_{\alpha \delta}\right)\right) .
\end{aligned}
$$

It follows from Assumption $\mathrm{H}_{1}$ (ii) and from $\left\|\alpha p_{\alpha \delta}\right\| \leq K r(\alpha) \rightarrow 0$ that $A_{\alpha \delta} \rightarrow 0$ uniformly with respect to $\delta<\alpha$. From $\mathrm{H}_{1}(\mathrm{i})$ we have $C_{\alpha \delta} \rightarrow 0$ as $\delta \rightarrow 0$ for each $\alpha$.

$B_{\alpha \delta}$ needs special consideration. It follows from $\mathrm{H}_{1}$ (iii) that

$$
B_{\alpha \delta} \geq-C\left\|\left(u_{\left\langle t_{\alpha \delta}, x_{\alpha \delta}\right\rangle}-v_{\left\langle s_{\alpha \delta}, y_{\alpha \delta}\right\rangle}\right)^{+}\right\|_{D}
$$

since for $(r, z) \in D$,

$$
\left(u\left(t_{\alpha \delta}+r, x_{\alpha \delta}+z\right)-v\left(s_{\alpha \delta}+r, y_{\alpha \delta}+z\right)\right)^{+} \leq \bar{M}^{+}\left(t_{\alpha \delta}\right)+\omega_{v}(\alpha) .
$$

Thus $B_{\alpha \delta} \geq-C \bar{M}^{+}\left(t_{\alpha \delta}\right)-C \omega_{v}(\alpha)$.

Letting $\alpha, \beta \rightarrow 0$ we finally obtain

$$
\eta^{\prime}\left(t_{0}\right) \leq C \bar{M}^{+}\left(t_{0}\right) .
$$

Since in case $\eta^{\prime}\left(t_{0}\right)=0$ the inequality (10) is obvious, we can apply Proposition 1 to obtain

$$
M(t) \leq M\left(t_{*}\right)+\int_{t_{*}}^{t} C \bar{M}^{+}(s) d s \quad \text { for } t_{*} \leq t \leq T
$$

and in view of $(4),(5)$,

$$
\bar{M}^{+}(t) \leq M_{0}^{+}\left(t_{*}\right)+\int_{t_{*}}^{t} C \bar{M}^{+}(s) d s .
$$

Hence Gronwall's inequality yields

$$
\bar{M}^{+}(t) \leq M_{0}^{+}\left(t_{*}\right) \exp (C t)
$$

and putting $t=T, M_{0}^{+}\left(t_{*}\right) \leq M_{0}^{+}(T)$ shows $M^{+}(T) \leq M_{0}^{+}(T) \exp (C T)$, which completes the proof.

For the case $(*)$ we will use the following lemma.

Lemma 1. Let $f \in \operatorname{BUC}(G)$ where $G \subset \mathbb{R}^{m}$ is an open domain, $\mu \in$ $C^{1}(G)$ and suppose $f-\mu$ attains a local maximum at some point $x_{0} \in G$. Then

$$
\left\|D \mu\left(x_{0}\right)\right\| \leq \limsup _{\lambda \rightarrow 0} \omega_{f}(\lambda) / \lambda .
$$

Proof. Suppose that $D \mu\left(x_{0}\right) \neq 0$. Put $h=-\left\|D \mu\left(x_{0}\right)\right\|^{-1} D \mu\left(x_{0}\right)$. Let $\lambda_{0} \geq \lambda>0$ be such that

$$
f\left(x_{0}+\lambda h\right)-\mu\left(x_{0}+\lambda h\right) \leq f\left(x_{0}\right)-\mu\left(x_{0}\right) .
$$

Define

$$
\varrho(\lambda)=\left[\mu\left(x_{0}+\lambda h\right)-\mu\left(x_{0}\right)-\lambda D \mu\left(x_{0}\right) h\right] \lambda^{-1} .
$$


Since $\mu\left(x_{0}+\lambda h\right)-\mu\left(x_{0}\right) \geq f\left(x_{0}+\lambda h\right)-f\left(x_{0}\right)$ we have

$$
\lambda \varrho(\lambda) \geq f\left(x_{0}+\lambda h\right)-f\left(x_{0}\right)-\lambda D \mu\left(x_{0}\right) h .
$$

As $D \mu\left(x_{0}\right) h=-\left\|D \mu\left(x_{0}\right)\right\|$ and $\|h\|=1$ we get

$$
\left\|D \mu\left(x_{0}\right)\right\| \leq \varrho(\lambda)+\left[f\left(x_{0}\right)-f\left(x_{0}+\lambda h\right)\right] \lambda^{-1} \leq \varrho(\lambda)+\omega_{v}(\lambda) / \lambda .
$$

Letting $\lambda \rightarrow 0$ we obtain (11).

Proof of Theorem 1(*). According to Lemma $1, D_{x} \lambda\left(t_{\alpha \delta}, x_{\alpha \delta}\right) \leq L$ where $L$ is a Lipschitz constant for $u$. Thus to deduce that $A_{\alpha \delta} \rightarrow 0$ the condition $\mathrm{H}_{1}(\mathrm{i})$ is sufficient.

3. The boundary value problem. In this section we investigate the problem (D). The following assumption will be needed.

Assumption $\mathrm{H}_{2}$. (i) The function $H: \Omega \times K_{1} \times K_{2} \times K_{3} \rightarrow \mathbb{R}$ is uniformly continuous for any bounded sets $K_{1} \subset \mathbb{R}, K_{2} \subset C(B), K_{3} \subset \mathbb{R}^{n}$.

(ii) For every $R \geq 0$,

$\lim _{\alpha \rightarrow 0} \sup \{|H(x, r, w, p)-H(y, r, w, p)|$ :

$$
|x-y|<\alpha,\left|x-y\|p \mid<\alpha,\| w \|_{B} \leq R\right\}=0 .
$$

(iii) There exist constants $L_{2}>L_{1}>0$ such that

$$
\begin{aligned}
& H(x, r, w, p)-H(x, r, \bar{w}, p) \geq-L_{1}\left\|(w-\bar{w})^{+}\right\|_{B}, \\
& H(x, r, w, p)-H(x, \bar{r}, w, p) \geq L_{2}(r-\bar{r}) \quad \text { for } r \geq \bar{r} .
\end{aligned}
$$

THEOREM 2. Suppose that Assumption $\mathrm{H}_{2}$ holds. Let $u, v \in \operatorname{BUC}\left(\Omega_{\tau}\right)$, $u \in \operatorname{SUB}\left(\Omega_{\tau}, f\right), v \in \operatorname{SUP}\left(\Omega_{\tau}, g\right)$ and $f, g \in C\left(\partial_{0} \Omega\right)$. Then

$$
\left\|(u-v)^{+}\right\|_{\Omega_{\tau}} \leq\left\|(f-g)^{+}\right\|_{\partial_{0} \Omega} .
$$

(*) If $u, v \in W^{1 \infty}\left(\Omega_{\tau}\right)$ then the condition (ii) of Assumption $\mathrm{H}_{2}$ is not necessary.

Pr o of. Since the proof is similar to that in Section 2 we will not repeat the details. Moreover, we will only consider the case when $\Omega$ is bounded.

Let $\mathcal{M}>0$, and let $\psi_{\alpha}$ be defined as in the proof of Theorem 1. Put

$$
\Psi(x, y)=u(x)-v(y)+5 \mathcal{M} \psi_{\alpha}(x-y) \quad \text { for } x, y \in \Omega .
$$

Let $\left(x_{\alpha}, y_{\alpha}\right) \in \Omega \times \Omega$ be such that $\sup \Psi=\Psi\left(x_{\alpha}, y_{\alpha}\right)$. Arguing as in Section 2 we obtain $\left\|x_{\alpha}-y_{\alpha}\right\| \leq \alpha$ and $\left\|x_{\alpha}-y_{\alpha}\right\| \leq \alpha r(\alpha)$ where $r(\alpha) \rightarrow 0$.

Suppose that $\left(x_{\alpha}, y_{\alpha}\right) \notin \Omega \times \Omega$ for some subsequence of $\alpha$. We can assume that $x_{\alpha} \in \partial \Omega$ (or $y_{\alpha} \in \partial \Omega$ ) and since $\Psi(x, x) \leq \Psi\left(x_{\alpha}, y_{\alpha}\right)$ for every $x \in \Omega$, in view of (13) we obtain

$$
u(x)-v(x) \leq u\left(x_{\alpha}\right)-v\left(y_{\alpha}\right)
$$


and

$$
u(x)-v(x) \leq u\left(x_{\alpha}\right)-v\left(x_{\alpha}\right)+v\left(x_{\alpha}\right)-v\left(y_{\alpha}\right) \leq\left\|(f-g)^{+}\right\|_{\partial_{0} \Theta}+\omega_{v}(\alpha),
$$

which, letting $\alpha \rightarrow 0$, implies (12).

Suppose now that $\left(x_{\alpha}, y_{\alpha}\right) \in \Omega \times \Omega$. Let

$$
\gamma(x)=v\left(y_{\alpha}\right)-5 \mathcal{M} \psi_{\alpha}\left(x-y_{\alpha}\right) \quad \text { and } \quad \lambda(y)=u\left(x_{\alpha}\right)+5 \mathcal{M} \psi_{\alpha}\left(x_{\alpha}-y\right) .
$$

Then $u-\gamma$ attains a local maximum at $x_{\alpha}$, and $v-\lambda$ attains a local minimum at $y_{\alpha}$.

As in the proof of Theorem 1 we obtain

$$
A_{\alpha}+B_{\alpha}+C_{\alpha} \leq 0
$$

where

$$
\begin{aligned}
& A_{\alpha}=H\left(x_{\alpha}, u\left(x_{\alpha}\right), u_{\left\langle x_{\alpha}\right\rangle}, p_{\alpha}\right)-H\left(y_{\alpha}, u\left(x_{\alpha}\right), u_{\left\langle x_{\alpha}\right\rangle}, p_{\alpha}\right), \\
& B_{\alpha}=H\left(y_{\alpha}, u\left(x_{\alpha}\right), u_{\left\langle x_{\alpha}\right\rangle}, p_{\alpha}\right)-H\left(y_{\alpha}, v\left(y_{\alpha}\right), u_{\left\langle x_{\alpha}\right\rangle}, p_{\alpha}\right), \\
& C_{\alpha}=H\left(y_{\alpha}, v\left(y_{\alpha}\right), u_{\left\langle x_{\alpha}\right\rangle}, p_{\alpha}\right)-H\left(y_{\alpha}, v\left(y_{\alpha}\right), v_{\left\langle y_{\alpha}\right\rangle}, p_{\alpha}\right)
\end{aligned}
$$

and $p_{\alpha}=D \gamma\left(x_{\alpha}\right)=D \lambda\left(y_{\alpha}\right)$.

Arguing as in Section 2 we find $A_{\alpha} \rightarrow 0$.

We can assume that $u\left(x_{\alpha}\right)-v\left(y_{\alpha}\right)>0$. (Otherwise (14) implies (12).) Notice that in view of $\mathrm{H}_{2}$ (iii) and (14),

$$
B_{\alpha} \geq L_{2}\left[u\left(x_{\alpha}\right)-v\left(y_{\alpha}\right)\right] \geq L_{2}\left\|(u-v)^{+}\right\|_{\Omega}
$$

and

$$
C_{\alpha} \leq L_{1}\left\|\left(u_{\left\langle x_{\alpha}\right\rangle}-v_{\left\langle y_{\alpha}\right\rangle}\right)^{+}\right\|_{B} \leq L_{1}\left\|\left(u_{\left\langle x_{\alpha}\right\rangle}-v_{\left\langle x_{\alpha}\right\rangle}\right)^{+}\right\|_{B}+L_{1} \omega_{v}(\alpha)-A_{\alpha},
$$

which gives

$$
L_{2}\left\|(u-v)^{+}\right\|_{\Omega} \leq L_{1}\left\|(u-v)^{+}\right\|_{\Omega_{\tau}}+L_{1} \omega_{v}(\alpha)-A_{\alpha} .
$$

If $\left\|(u-v)^{+}\right\|_{\Omega_{\tau}} \leq\left\|(u-v)^{+}\right\|_{\Omega}$, (16) gives a contradiction $(\alpha \rightarrow 0)$ since $L_{1}>L_{2}$. Hence $\left\|(u-v)^{+}\right\|_{\Omega_{\tau}}>\left\|(u-v)^{+}\right\|_{\Omega}$, which implies (12). The proof is complete.

The case $(*)$ is treated in the same way as in Theorem 1 .

4. "Almost everywhere" solutions. To end our considerations, we present an application of our results to the theory of a.e. solutions.

We will treat the problem (D) ((C) can be treated in the same way). Moreover, without loss of generality we can assume that $H$ does not depend on $r$.

Let $G \subset \mathbb{R}^{n}$ be an open domain. Define

$$
G(\varrho)=\{x \in G: \operatorname{dist}(x, \partial G)>\varrho\} \quad \text { for } \varrho>0 .
$$


Definition 2. A function $u \in C(G)$ is said to be semiconcave if for every $\varrho>0$ there exists a constant $C_{\varrho} \in \mathbb{R}$ such that $u(x)-C_{\varrho}\|x\|^{2} / 2$ is concave on every convex subset of $G(\varrho) \cap B(0,1 / \varrho)$.

TheOREM 3. Let $H(x, w, p)$ be continuous, $u \in W_{\operatorname{loc}}^{1 \infty}\left(\Omega_{\tau}\right)$, and $g \in$ $C\left(\partial_{0} \Omega\right)$.

(i) If $H(x, w, p)$ is convex in $p$, satisfies $H\left(x, u_{\langle x\rangle}, D u(x)\right) \leq 0$ a.e. in $\Omega$, and $u \leq g$ in $\partial_{0} \Omega$ then $u \in \operatorname{SUB}\left(\Omega_{\tau}, g\right)$.

(ii) If $u$ is semiconcave, satisfies $H\left(x, u_{\langle x\rangle}, D u(x)\right) \geq 0$ a.e. in $\Omega$, and $u \geq g$ in $\partial_{0} \Omega$ then $u \in \operatorname{SUP}\left(\Omega_{\tau}, g\right)$.

The proof presented below is a generalization of the proof of Proposition III.3 in [3].

In the proof of (i) we will use the following lemma.

LEMMA 2. Let $F_{k}, F \in C\left(\Omega \times C(B) \times \mathbb{R}^{n}\right), g_{k}, g \in C\left(\partial_{0} \Omega\right)$, and $F_{k} \rightarrow F$, $g_{k} \rightarrow g$. Suppose that $u_{k} \in \operatorname{SUB}\left(\Omega_{\tau}, F_{k}, g_{k}\right)$ (resp. $\left.u_{k} \in \operatorname{SUP}\left(\Omega_{\tau}, F_{k}, g_{k}\right)\right)$ and $u_{k} \rightarrow u$ in $C\left(\Omega_{\tau}\right)$. Then $u \in \operatorname{SUB}\left(\Omega_{\tau}, F, g\right)$ (resp. $\left.u \in \operatorname{SUP}\left(\Omega_{\tau}, F, g\right)\right)$.

P r o of. Suppose that $u-\varphi$ attains a local maximum at $x_{0} \in \Omega$. We can assume (see [3], Lemma 1.4) that $(u-\varphi)(x)<(u-\varphi)\left(x_{0}\right)$ for $x \in \Omega, x \neq x_{0}$. Let $\delta>0$ be such that $x_{0} \in \Omega(\delta)$. Put $x_{k}=\sup \left\{\left(u_{k}-\varphi\right)(x): x \in \Omega(\delta)\right\}$. Without loss of generality we can assume that $x_{k} \rightarrow \bar{x} \in \overline{\Omega(\delta)}$. We claim that $x_{0}=\bar{x}$. Indeed, since $\left(u_{k}-\varphi\right)\left(x_{0}\right) \leq\left(u_{k}-\varphi\right)\left(x_{k}\right)$, letting $k \rightarrow \infty$ we obtain $(u-\varphi)\left(x_{0}\right) \leq(u-\varphi)(\bar{x})$ and thus $x_{0}=\bar{x} \in \Omega(\delta)$. We can assume (taking subsequences if necessary) that $x_{k} \in \Omega(\delta)$, which implies that $u_{k}-\varphi$ attains a local maximum at $x_{k} \in \Omega(\delta)$. Then by assumption $F_{k}\left(x_{k}, u_{\left\langle x_{k}\right\rangle}, D \varphi\left(x_{k}\right)\right) \leq 0$ and letting $x_{k} \rightarrow x_{0}, F\left(x_{0}, u_{\left\langle x_{0}\right\rangle}, D \varphi\left(x_{0}\right)\right) \leq 0$, which completes the proof, since the boundary inequality is obvious.

Now we will prove the theorem.

Proof of (i). Let $u \in W_{\text {loc }}^{1 \infty}\left(\Omega_{\tau}\right)$ and suppose $H\left(x, u_{\langle x\rangle}, D u(x)\right) \leq 0$ a.e. in $\Omega$. Put $u^{\varepsilon}=u * p_{\varepsilon}$ where $p_{\varepsilon}=p(\cdot / \varepsilon) \varepsilon^{n}, p \in C^{\infty}\left(\mathbb{R}^{n}\right), p \geq 0$, $\operatorname{supp} p \subseteq B(0,1), \int_{\mathbb{R}^{n}} p d \mu=1, \varepsilon>0$. We have $u^{\varepsilon} \in C^{1}\left(\Omega_{\tau}(\delta)\right), \Omega_{\tau}(\delta) \subseteq \Omega_{\tau}$ and $u_{\varepsilon} \rightarrow u$ in $\Omega_{\tau}(\delta)$ for $\varepsilon<\delta$. Notice that, since

$$
H\left(x, u_{\langle x\rangle}^{\varepsilon}, D u^{\varepsilon}(x)\right) \leq H\left(x, u_{\langle x\rangle}^{\varepsilon}, D u^{\varepsilon}(x)\right)-H\left(x, u_{\langle x\rangle}, D u(x)\right) \quad \text { in } \Omega(\delta),
$$

we have

$$
H\left(x, u_{\langle x\rangle}^{\varepsilon}, D u^{\varepsilon}(x)\right) \leq A_{\varepsilon}(x)+B_{\varepsilon}(x) \quad \text { in } \Omega(\delta)
$$

where

$$
A_{\varepsilon}(x)=H\left(x, u_{\langle x\rangle}^{\varepsilon}, D u^{\varepsilon}(x)\right)-H\left(x, u_{\langle x\rangle}, D u^{\varepsilon}(x)\right)
$$




$$
B_{\varepsilon}(x)=H\left(x, u_{\langle x\rangle}, D u(x)\right) * p_{\varepsilon}(x)-H\left(x, u_{\langle x\rangle}, D u(x)\right) .
$$

In the last inequality we based on the fact that for $H$ convex in $p, H\left(x, u_{\langle x\rangle}\right.$, $\left.D u^{\varepsilon}(x)\right) \leq H\left(x, u_{\langle x\rangle}, D u(x)\right) * p_{\varepsilon}(x)$. Notice that $A_{\varepsilon}, B_{\varepsilon} \rightarrow 0$ in $\Omega(\delta)$.

Since $u^{\varepsilon}$ is a viscosity subsolution of (16) with boundary function $g_{\varepsilon}=u^{\varepsilon}$ in $\Omega_{\tau}(\delta) \backslash \Omega(\delta)$, in view of Lemma 2 (taking $\Omega(\delta)$ instead of $\Omega$ ) letting $\delta \rightarrow 0$ we conclude that $u \in \operatorname{SUB}\left(\Omega_{\tau}, g\right)$, which completes the proof of (i).

Proof of (ii). Let $\varphi \in C^{1}(\Omega)$. Assume that $u-\varphi$ attains a local minimum at some point $x_{0} \in \Omega$. Let $\delta>0$ be such that $x_{0} \in \Omega(\delta)$. Without loss of generality we can assume that $u$ is concave in $\Omega(\delta)$ (otherwise we consider $\left.\bar{H}(x, w, p)=H\left(x, w+\left(C_{\delta} / 2\right)|\cdot|_{\langle x\rangle}^{2}, p+C_{\delta}|x|\right)\right)$.

Since $u(x) \geq u\left(x_{0}\right)-\varphi\left(x_{0}\right)+\varphi(x)$ in some ball $B\left(x_{0}, \varrho\right)$,

$$
u(x)-u\left(x_{0}\right)-D \varphi\left(x_{0}\right)\left(x-x_{0}\right) \geq \beta(x)\left\|x-x_{0}\right\|
$$

where $\beta(x) \rightarrow 0$ as $x \rightarrow x_{0}$.

Put $x_{1}=2 x_{0}-x$. Since $u$ is concave, $u(x) \leq 2 u\left(x_{0}\right)-u\left(x_{1}\right)$ and

$$
\begin{aligned}
u(x)-u\left(x_{0}\right)-D \varphi\left(x_{0}\right)\left(x-x_{0}\right) & \leq u\left(x_{0}\right)-u\left(x_{1}\right)-D \varphi\left(x_{0}\right)\left(x_{0}-x_{1}\right) \\
& \leq \varphi\left(x_{0}\right)-\varphi\left(x_{1}\right)-D \varphi\left(x_{0}\right)\left(x_{0}-x_{1}\right) \\
& \leq \bar{\beta}(x)\left\|x-x_{0}\right\|
\end{aligned}
$$

where $\bar{\beta}(x) \rightarrow 0$ as $x \rightarrow x_{0}$. This inequality and (18) imply that $u$ is differentiable at $x_{0}$ and $D u\left(x_{0}\right)=D \varphi\left(x_{0}\right)$.

Since, by assumption, there exist $\left\{x_{m}\right\} \in \Omega(\delta), x_{m} \rightarrow x_{0}, u$ is differentiable at $x_{m}$ and $H\left(x_{m}, u_{\left\langle x_{m}\right\rangle}, D u\left(x_{m}\right)\right) \geq 0$. We claim that for every sequence $x_{m} \rightarrow x_{0}, D u\left(x_{m}\right) \rightarrow D u\left(x_{0}\right)$. Indeed, suppose that $p=$ $\lim _{k \rightarrow \infty} D u\left(x_{m\langle k\rangle}\right)$ and $\left(p-D u\left(x_{0}\right)\right)_{i} \neq 0$ for some $i \in\{1, \ldots, n\}$. Put $\bar{x}=x_{0}+h \operatorname{sgn}\left(p-D u\left(x_{0}\right)\right)_{i} e_{i}, e_{i} \in \mathbb{R}^{n},\left(e_{i}\right)_{j}=\delta_{i j}, j=1, \ldots, n, h>0$. Since $D u\left(x_{m}\right)$ is uniformly bounded and $u$ is concave we have

$$
\left[u(\bar{x})-u\left(x_{0}\right)-p\left(\bar{x}-x_{0}\right)\right] /\left\|\bar{x}-x_{0}\right\| \leq 0,
$$

which implies

$$
\left[u(\bar{x})-u\left(x_{0}\right)-D u\left(x_{0}\right)\right] /\left\|\bar{x}-x_{0}\right\|+\left|\left(D u\left(x_{0}\right)-p\right)_{i}\right| \leq 0 .
$$

Letting $h \rightarrow 0$ we obtain $\left(D u\left(x_{0}\right)-p\right)_{i}=0$, which contradicts our assumption. Since $D u\left(x_{m}\right)$ is uniformly bounded, this means that $D u\left(x_{m}\right) \rightarrow$ $D u\left(x_{0}\right)$.

As $u_{\left\langle x_{m}\right\rangle} \rightarrow u_{\left\langle x_{0}\right\rangle}$ in $C(B)$ and $D u\left(x_{0}\right)=D \varphi\left(x_{0}\right)$ we conclude letting $n \rightarrow \infty$ that $H\left(x_{0}, u_{\left\langle x_{0}\right\rangle}, D u\left(x_{0}\right)\right) \geq 0$ and thus $u \in \operatorname{SUP}\left(\Omega_{\tau}, g\right)$. The proof is complete. 


\section{References}

[1] A. Augustynowicz and Z. Kamont, On Kamke's functions in uniqueness theorems for first order partial differential-functional equations, Nonlinear Anal. 14 (1990), 837-850.

[2] M. G. Crandall, L. C. Evans and P. L. Lions, Some properties of viscosity solutions of Hamilton-Jacobi equations, Trans. Amer. Math. Soc. 282 (1984), 487502.

[3] M. G. Crandall and P. L. Lions, Viscosity solutions of Hamilton-Jacobi equations, ibid. 277 (1983), 1-42.

[4] Z. Kamont, On the Cauchy problem for system of first order partial differentialfunctional equations, Serdica 5 (1979), 327-339.

[5] S. N. Kruzhkov, Generalized solutions of nonlinear first order partial differential equations, Mat. Sb. 70 (1966), 394-415 (in Russian).

[6] -, Generalized solutions of the Hamilton-Jacobi equations of eikonal type. I, ibid. 98 (1975), 450-493 (in Russian).

[7] V. Lakshmikantham and S. Leela, Differential and Integral Inequalities, Academic Press, New York, 1969.

[8] H. Leszczyński, A contribution to the uniqueness theory for first-order partial differential-functional systems, Dissertationes Math., to appear.

[9] P. L. Lions, Generalized Solutions of Hamilton-Jacobi Equations, Pitman, London, 1982.

[10] J. Szarski, Differential Inequalities, PWN, Warszawa, 1967.

INSTITUTE OF MATHEMATICS

UNIVERSITY OF GDAŃSK

WITA STWOSZA 57

80-952 GDAŃSK, POLAND 